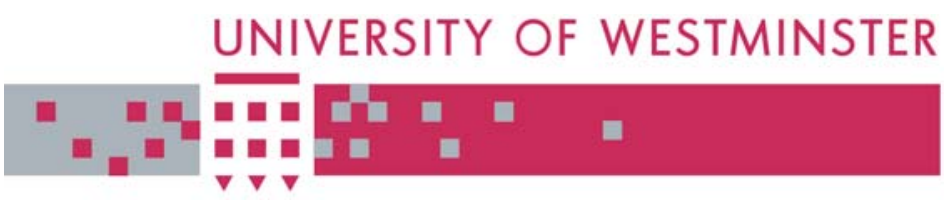

\title{
WestminsterResearch
}

http://www.wmin.ac.uk/westminsterresearch

\section{Low intermodulation amplifiers for RF and microwave wireless systems.}

\author{
Natasa Males-Ilic ${ }^{1}$ \\ Bratislav Milovanovic ${ }^{2}$ \\ Djuradj Budimir ${ }^{1}$
}

${ }^{1}$ School of Informatics, University of Westminster

${ }^{2}$ Faculty of Electronic Engineering, University of Nis

Copyright (C [2001] IEEE. Reprinted from the proceedings of the 2001 AsiaPacific Microwave Conference, 2001. APMC 2001, pp. 984-987.

This material is posted here with permission of the IEEE. Such permission of the IEEE does not in any way imply IEEE endorsement of any of the University of Westminster's products or services. Internal or personal use of this material is permitted. However, permission to reprint/republish this material for advertising or promotional purposes or for creating new collective works for resale or redistribution must be obtained from the IEEE by writing to pubs-permissions@ieee.org. By choosing to view this document, you agree to all provisions of the copyright laws protecting it.

The WestminsterResearch online digital archive at the University of Westminster aims to make the research output of the University available to a wider audience. Copyright and Moral Rights remain with the authors and/or copyright owners.

Users are permitted to download and/or print one copy for non-commercial private study or research. Further distribution and any use of material from within this archive for profit-making enterprises or for commercial gain is strictly forbidden.

Whilst further distribution of specific materials from within this archive is forbidden, you may freely distribute the URL of the University of Westminster Eprints (http://www.wmin.ac.uk/westminsterresearch).

In case of abuse or copyright appearing without permission e-mail wattsn@wmin.ac.uk. 


\title{
LOW INTERMODULATION AMPLIFIERS FOR RF and MICROWAVE WIRELESS SYSTEMS
}

\author{
NATASA MALES-ILIC, \\ Wireless Communication Research Group, Department of Electronic Systems, \\ University of Westminster, \\ 1.15 New Cavendish Street London W1W 6UW UK, \\ E-mail: males-ilic@cmsa.wmin.ac.uk \\ BRATISLAV MILOVANOVIC \\ Faculty of Electronic Engineering, University of Nis, Beogradska 14, 18000 Nis, Yugoslavia, \\ E-mail: bata@elfak.ni.ac.yu \\ DJURADJ BUDIMIR, \\ Wireless Communication Research Group, Department of Electronic Systems, \\ University of Westminster, \\ 115 New Cavendish Street London W1W 6UW UK, \\ E-mail: budimid@wmin.ac.uk
}

\begin{abstract}
A novel linearisation technique for reduction in the third-order intermodulation distortion products, with the injection of the second harmonics through a feedback loop of a power amplifier was applied in this paper. The power amplifier including the feedback loop components (bandpass filter, phase shifter, attenuator) was designed as a hybrid microwave integrated circuit by using program Libra. The adjustable parameters are the phase and amplitude of the loop signals. Therefore, a voltage that controls a phase shift of the phase shifter and a control current of a PIN diode in the attenuator circuit were optimised to obtain a reduction in the third-order intermodulation distortion. The achievable improvement was found to be $21 \mathrm{~dB}$ for the case of two fundamental signals at the power amplifier input.
\end{abstract}

\section{$1 \quad$ Introduction}

In telecommunications systems, the intermodulation distortion (IMD) especially the third-order (IMD3) generated in-band, has always been of concern, particularly when many multilevel channels are simultaneously processed. Many different techniques for IMD reduction can be found in literature such as predistortion, feedforward, feedback and combination of them [1-2]. However, the application of these techniques requires the circuitry that may be complex, expensive and large in size, as well as limits the usage of active device full capability. In a novel technique for reducing the IMD product levels $[3,4]$, the second harmonics of the input signals are fed together with the fundamental signal to the amplifier input. The injection of the difference frequency between the fundamental signals is another way to reduce IMD product levels [5]. Both approaches of a novel technique satisfy the reduction of IMD product levels without affecting the fundamental signal power levels. This work extends previous analyses of a multicarrier amplification. The effects of the injection of carrier second harmonics on the intermodulation in a microwave power amplifier were investigated. The published results preceding this paper is based on the approach in which feedback loop components were modeled by ideal elements from the library of commercial programs such as Libra or MDS. In our work, second harmonics are extracted from a non-linear power amplifier output, and returned to the amplifier input through the feedback loop, whose components are band pass filter, phase shifter, attenuator and isolator. Each of them, excepting the isolator, was designed for the application in a hybrid MIC of power amplifier. 
The power amplifier circuit with feedback loop is presented in Fig. 1. A design was applied on the substrate characterised by following parameters $\varepsilon_{r}=4.3, H=0.635 \mathrm{~mm}, t=0.004 \mathrm{~mm}$. In power amplifier simulation NEC MESFET from Libra library denoted as NE710 was used.

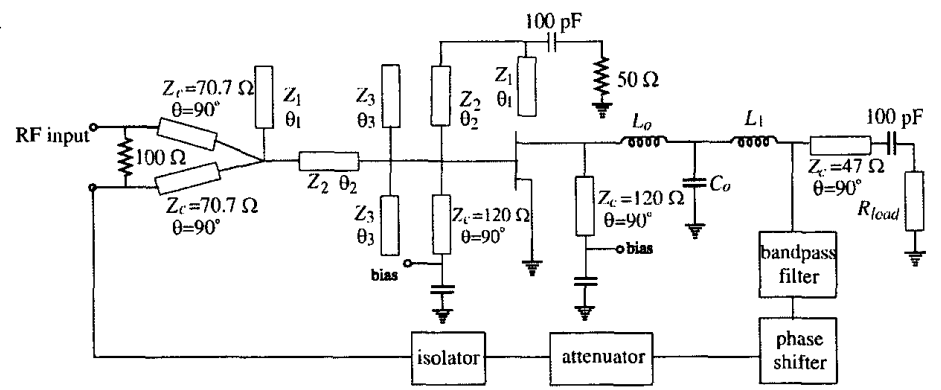

Fig.1. Power amplifier with the second harmonics feedback loop

The proposed technique uses the amplifier non-linear characteristic to generate a second third-order IMD signal which is used to cancel the original third-order IMD product at the output. Namely, by proper selection of phase and amplitude of the second harmonics, it is possible to make the third-order IMD product produced by the second harmonics out of phase and equal in the amplitude with the original third-order product. With the aim to select only the second harmonic components at the output of the power amplifier, the first device in the feedback loop must be a bandpass filter. The bandpass filter that can be conveniently fabricated in microstrip is the capacitiv-gap coupled resonator filter designed at $5.3 \mathrm{GHz}$ centre frequency with $20 \%$ bandwidth and $0.5 \mathrm{~dB}$ equal-ripple response, with 3 sections. A $360^{\circ}$ reflection-type analogue phase shifter with a single $90^{\circ}$ branch line coupler [6] was designed. The phase shifter circuitry is shown in Fig. 2. The phase shift in this phase shifter is produced by reflecting the incident wave with a varactor diode, whose capacitance varies according to the bias voltage. As insertion loss strongly depends on impedance $Z_{1}$ (Fig. 2), its appropriate value to the reflection load (two varactors in series with a shorted stub and separated by quarterwave transmission line) is provided by the impedance transformer. GaAs hyperabrupt tuning varactor type MA-46553 was used for simulation. Varying the controlled voltage between 2 and $20 \mathrm{~V}$, a full phase shift of $360^{\circ}$ is achieved at the frequencies of the fundamental signal second harmonics. An appropriate attenuation of the second harmonics is accomplished by PIN diode attenuator shown in Fig. 3. For simulation purposes, HP hermetic PIN diode for microstrip attenuators was used. Changing a diode forward current from 0.01 to $100 \mathrm{~mA}$, the resistance of the intrinsic region of the diode is varied, providing the control of attenuation with the bias point. The input and output of the attenuator are at the input and isolated ports of the $3 \mathrm{~dB}$ Lange-coupler selected in order to attain better performances over wider frequency range. Simulated results show that the attenuation controlled by the bias current varies from $0.5-33 \mathrm{~dB}$.

Returned signals of the second harmonics are combined with fundamental input signals over Wilkinson's combiner, Fig. 1.

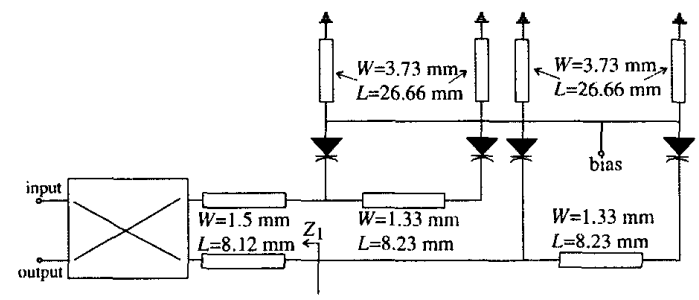

Fig.2. Phase shifter

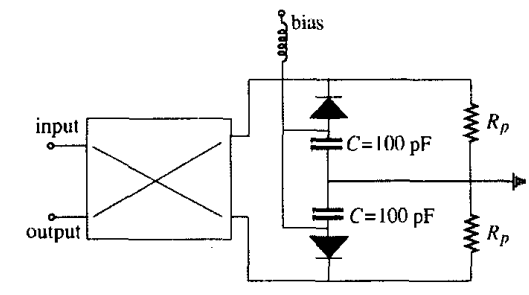

Fig.3. Variable attenuator 


\section{$3 \quad$ Numerical and Experimental Results}

The realtiv phase shift characteristics of the phase shifter over the frequency range $5-5.2 \mathrm{GHz}$ for the control voltage values of 4, 9, 14 and $20 \mathrm{~V}$ denoted as PH-control voltage are shown in Fig. 4 . The attenuation characteristics of variable attenuator in the frequency range 5-5.4 GHz, that relate to PIN diode intrinsic resistance of 1,50,200 and $500 \Omega$ are represented in Fig. 5, with an appropriate denotation ATEN-resistance value.

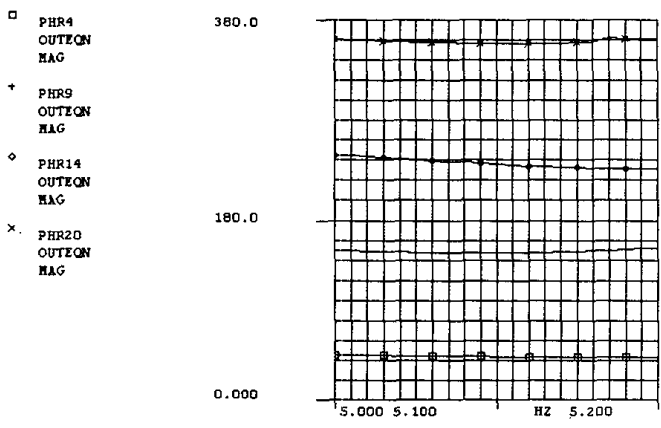

Fig. 4 Relativ phase shift of reflection type phase shifter

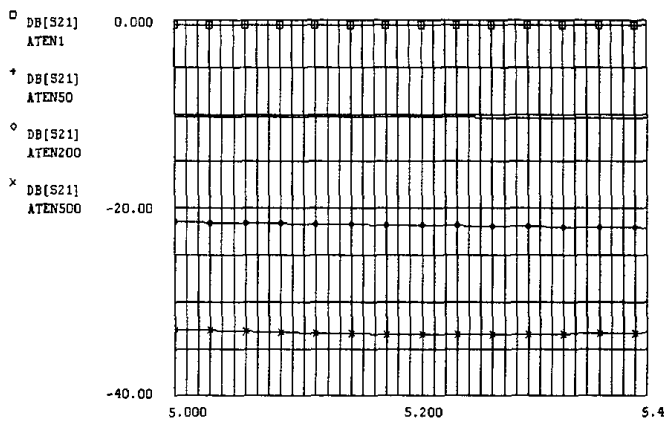

Fig. 5 Attenuation of variable atteniator

The chosen frequencies of two main input fundamental signals are $2.5 \mathrm{GHz}$ and $2.51 \mathrm{GHz}$ and their input power levels are $-2 \mathrm{dBm}$. In the CAD simulation, non-linear Curtice's cubic model was used for MESFET modeling. Input reflection coefficient less than $-10 \mathrm{~dB}$ in the frequency range $2-9 \mathrm{GHz}$ as well as unconditionally stable operation of MESFET were achieved by the proposed input matching circuit (Fig. 1). The values of $L_{0}, L_{1}, C_{0}$ and transformer impedance, Fig. 1, were changed by using optimisation facility of program Libra in order to accomplish desired performance of fundamental signals' output power of approximately $3 \mathrm{dBm}$ and the lowest third order IMD power of $-57 \mathrm{dBm}$. The spectrum for the bias point $V_{g s}=-0.4 \mathrm{~V}$ and $V_{d s}=3 \mathrm{~V}$, obtained at the output without applying our technique is shown in Fig. 6a). It includes fundamental signals and the third-order IMD products at $2.49 \mathrm{GHz}$ and $2.52 \mathrm{GHz}$. When the second harmonics were returned from the output to the input of the power amplifier, CAD optimisation was used to find the right phase and amplitude of these signals in order to reduce the IMD3 products by keeping the fundamental signal power levels constant. The spectrum obtained after simulation are shown in Fig. 6b).
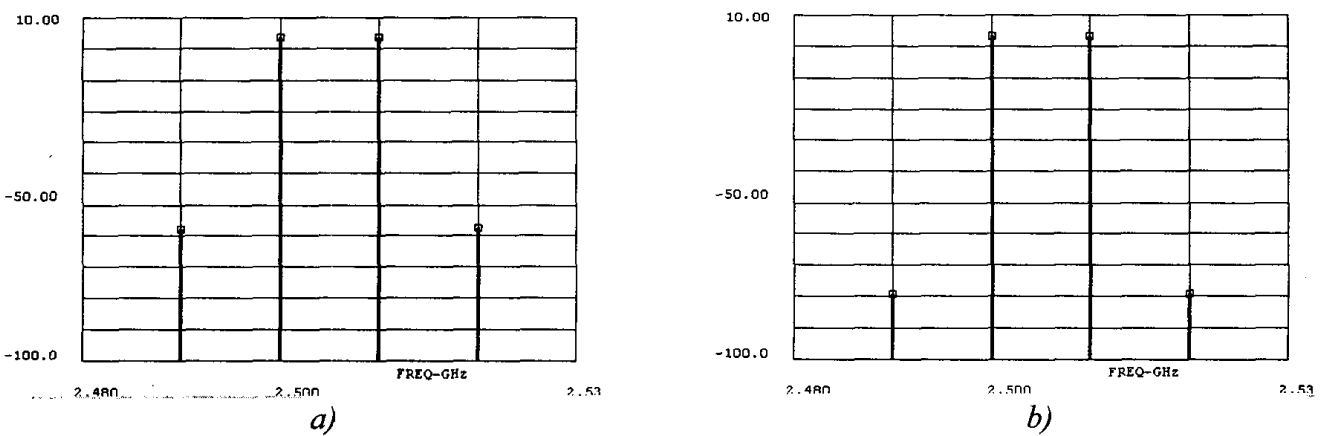

Fig. 6. The simulated fundamental powers and third order IMD powers a) Before employing the technique; b) After employing the technique

The results obtained during analyses show that a maximum reduction of approximately $40 \mathrm{~dB}$ of each IMD3 product can be obtained separately without reduction of the fundamental signals. The values of the phase shift and attenuation at which maximum reduction is obtained are different for the various IMD3 products. The second harmonic spectral components must have approximately the same power level so that all of them can be controlled to within a fraction of $\mathrm{dB}$ in amplitude and of a few degrees 
in phase in order to attain maximum reduction in IMD3 products. On the other hand, it is difficult to obtain a maximum reduction in all IMD3 products with the same amplitude and phase adjustment due to these products have slightly different amplitudes and phases. The results shown in Fig. 6 refer to the compromise between the maximum reductions in each IMD3 signal obtained separately, yielding 21 $\mathrm{dB}$ improvement in both. These results were achieved with phase shift of $14.5^{\circ}$ and attenuation of 0.9 $\mathrm{dB}$. The experimental results on a commercially available amplifier is plotted and shown in Figures $7 \mathrm{a}$ and $7 \mathrm{~b}$ respectively.

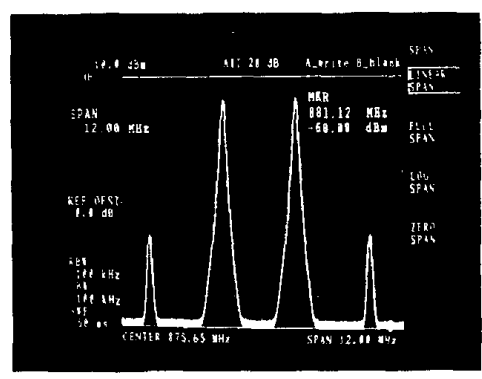

a)

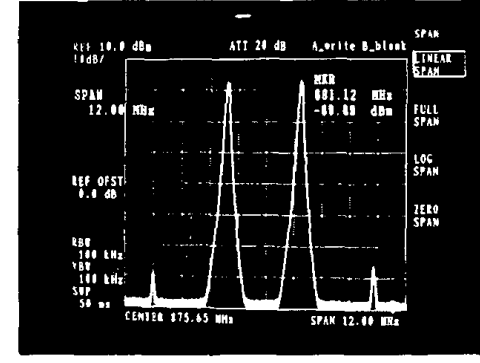

b)

Figure 7 The measured fundamental power and third order IM power for Pin $=-20 \mathrm{dBm}$ (a) before and (b) after employing the technique

\section{Conclusion}

In order to reduce the third-order intermodulation products of power amplifier, a novel linearisation technique was applied. The second harmonics of the fundamental signals were injected through the feedback loop of power amplifier. The proposed technique uses the amplifier non-linear characteristic to generate a second third-order IMD signal, which is used to cancel the original third-order IMD product at the output. The earlier published results referring to the same novel technique approach are based on the power amplifier simulation for the ideal feedback loop elements. In this paper the feedback loop components (band pass filter, phase shifter, attenuator) as well as a single stage amplifier circuit were designed as a hybrid microwave integrated circuit in a microstrip technique using program Libra. Adjusting the phase shift and amplitude of the loop signals; the reduction in third-order intermodulation distortion products obtained by simulation are $21 \mathrm{~dB}$ in both and obtained during experiment are approximately $17 \mathrm{~dB}$.

\section{References}

[1] J .G. Mc Rory, R.H. Johnson , "An RF Amplifier For Low IM Distortion " , IEEE MTT-S Digest ,pp. 1741 - 1744,1994 .

[2] Daniel Myer, “Design Linear Feedforward Amplifiers For PCN Systems ”, Design Feature, Microwaves \& $R F$, pp.121-133, September 1994.

[3] M.R. Moazzam, C.S. Aitchison, "The reduction of Third Order Intermodulation Product in Microwave Amplifiers", IEE Colloquium on "Solid State Power Amplification and Generation", Digest No:1996/013, pp.7/1-7/5, Savoy Place London, 25 January 1996.

[4] M.R. Moazzam, C.S. Aitchison, "A Low Third Order Intermodulation Amplifier With Harmonic Feedback Circuitry", IEEE MTT-S Digest, pp. 827-830, 1996.

[5] Budimir, D., M. Modeste, and C. S. Aitchison "A Difference Frequency Technique for Improving IM Performance of RF Amplifiers", Microwave and Optical Technology Letters, Vol. 24, No. 3, pp.208-210, February 2000

[6] T. Yoo, J. Song, M. Park, " $360^{\circ}$ reflection-type analogue phase shifter implemented with a single $90^{\circ}$ branch-line coupler", Electornics Letters, Vol.33 No. 3, pp.224-226, January 1997. 